
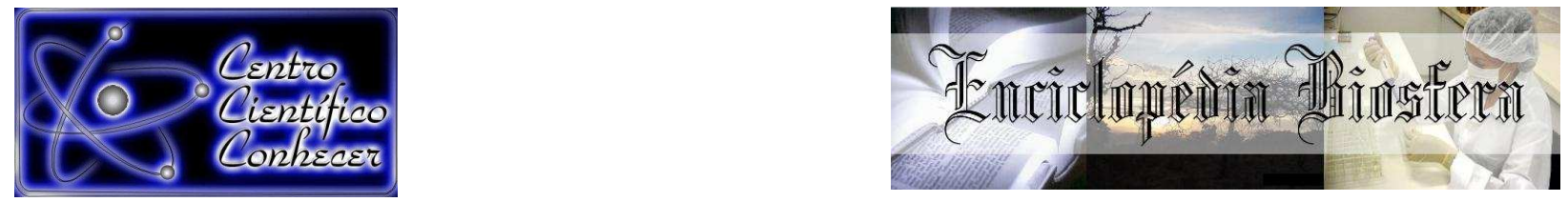

\title{
INFLUÊNCIA DO TIPO HISTOPATOLÓGICO E DO TEMPO DE EVOLUÇÃO NO TAMANHO DE NEOPLASIAS MAMÁRIAS EM CADELAS
}

William Torres Blanca1, Lígia Fernandes Gundim¹, Thais Almeida Moreira 2, Ednaldo Carvalho Guimarães ${ }^{3}$, Alessandra Aparecida Medeiros ${ }^{4}$

1 Mestrando do curso de pós-graduação em Ciências Veterinária da Universidade Federal de Uberlândia (wiliwiltb@hotmail.com), Uberlândia-Brasil.

2 Residente do Laboratório de Patologia Animal da Universidade Federal de Uberlândia.

3 Professor Doutor da Faculdade de Matemática da Universidade Federal de Uberlândia

4 Professora Doutora do setor Patologia Animal da Faculdade de Medicina Veterinária da Universidade Federal de Uberlândia.

Recebido em: 08/09/2015 - Aprovado em: 14/11/2015 - Publicado em: 01/12/2015 DOI: http://dx.doi.org/10.18677/Enciclopedia_Biosfera_2015_181

\begin{abstract}
RESUMO
Neoplasias da glândula mamária apresentam alta ocorrência em cadelas e possuem diversos fatores correlacionados ao seu prognóstico. Estudos sobre a correlação entre o tamanho e demais características dos tumores mamários, como o tipo histopatológico da neoplasia, demonstram-se controversos tanto na medicina humana quanto na veterinária. Dessa forma, objetivou-se verificar se há correlação entre o tempo de evolução da doença e o tamanho da neoplasia e ainda, se existe correlação entre o tamanho do tumor e a classificação histopatológica de neoplasias mamárias de cadelas. Para isso, foram utilizadas 79 cadelas com diagnóstico clínico de carcinoma mamário e coletadas informações sobre o tempo de evolução das neoplasias. As massas tumorais foram mensuradas em seu maior comprimento, e agrupadas conforme proposta da Organização Mundial de Saúde (OMS) em T1 (abaixo de $3 \mathrm{~cm}$ ), T2 (entre $3 \mathrm{~cm}$ a $5 \mathrm{~cm}$ ) e T3 (acima de $5 \mathrm{~cm}$ ). As correlações entre o tamanho da massa tumoral e o tempo de evolução foram realizados através do teste de Pearson. A correlação entre o tipo histopatológico e o tempo de evolução foi realizado utilizando o teste Chi-quadrado e Kruskal Wallis. Concluiu-se que não há correlação entre o tamanho e o tipo de carcinoma, o que difere de demais estudos recentes. Entretanto, observou-se fraca correlação entre o tamanho e o tempo de evolução destas neoplasias.
\end{abstract}

PALAVRAS-CHAVE: características tumorais, carcinomas, desenvolvimento tumoral.

\section{INFLUENCE OF HISTOPATHOLOGICAL TYPE AND EVOLUTION OF TIME IN SIZE IN BREAST NEOPLASMS IN FEMALE DOGS}

\begin{abstract}
Neoplasms of the mammary gland have high incidence in dogs and have many factors related to prognosis. Studies on the correlation between the size and other characteristics of breast tumors, as histopathological type of tumor, show up
\end{abstract}


controversial both in human medicine and in veterinary medicine. Thus, the objective was to verify if there is correlation between the progression of the disease and the size of the tumor and, if there is a correlation between tumor size and histopathological classification of breast tumors in female dogs. For this, we used 79 dogs with clinical diagnosis of breast carcinoma and collected information on the time course of neoplasms. The tumor masses were measured in their greater length and grouped together as proposed by the World Health Organization (WHO) T1 (less than $3 \mathrm{~cm}$ ), T2 (between 3 to $5 \mathrm{~cm}$ ) and T3 (above $5 \mathrm{~cm}$ ). The correlations between the size of the tumor mass and the time of evolution were performed using Pearson's test. The correlation between the pathological type and the time of evolution was performed using the Chi-square test and Kruskal Wallis. It was concluded that there is no correlation between the size and type of carcinoma, which differs from other recent studies. However, we noticed a weak correlation between the size and the time of evolution of these neoplasms.

KEYWORDS: tumor characteristics, carcinomas, duration.

\section{INTRODUÇÃO}

Os tumores mamários são as neoplasias mais frequentes em cadelas, sendo essa frequência, duas a três vezes superiores à observada na mulher. As neoplasias mamárias em caninos apresentam várias características epidemiológicas, clinicopatológicas, biológicas, bioquímicas e genéticas, semelhantes ao da espécie humana. Sendo assim, a correlação de estudos do câncer de mama na mulher com o tumor de mama na cadela tem sido explorada (PINHO et al., 2012; CASSALI et al., 2013).

Em cadelas, aproximadamente $50 \%$ dos casos destas neoplasias são de caráter maligno e em $25 \%$ dos casos apresentam metástases em linfonodos regionais e pulmões (De NARDI et al., 2007). Acometem cadelas adultas e idosas e todas as raças de cães, entretanto quando precocemente diagnosticadas, o prognóstico é favorável (DORÉ et al., 2003). A carcinogênese do tumor de mama em cadelas ainda não é totalmente elucidada, mas sabe-se que a etiologia é multifatorial, com participação de fatores genéticos, ambientais e hormonais (DORÉ et al., 2003).

O estadiamento clínico é determinado de acordo com o sistema TNM estabelecido pela Organização Mundial da Saúde (OMS) para tumores mamários caninos, que considera as características: tamanho do tumor primário $(T)$, o envolvimento de linfonodos regionais $(\mathrm{N})$ e a presença ou ausência de metástases à distância (M) (TNM, 1998; CASSALI, et al., 2013).

Com exceção o carcinoma inflamatório, recomenda-se como tratamento de tumores mamários, a cirurgia. A sobrevida não é influenciada pela técnica utilizada, desde que todo o tecido tumoral seja removido e a margem de segurança respeitada (MARQUES, 2013).

O tamanho dos tumores mamários foi considerado um fator independente para prognóstico, porém tumores menores que três centímetros estão associados a melhores prognósticos quando comparado a tumores maiores (CASSALI, et al., 2013). Tumores com maiores proporções estão relacionados ao número de células em multiplicação (MATOS, et al., 2012) e segundo SORENMO et al., (2009) quanto maior for o tumor, maior tendência à malignidade.

Já o tempo de evolução dos tumores mamários é extremamente variável na espécie canina e está ligado, dentre outros fatores, à malignidade do tumor (MATOS, et al., 2012). Segundo PETROV, et al. (2014) o diagnóstico tardio é um das 
principais causas de morte, pois está associado à metástases pulmonares. Para realização de um estudo sistemático é aconselhável a classificação dos carcinomas mamários em três tipos, sendo eles: carcinoma simples, quando há apenas proliferação epitelial; carcinoma complexo, quando apresenta proliferação mioepitelial e epitelial; e carcinoma em tumor misto quando possui proliferação epitelial com metaplasia cartilaginosa (KARAYANNOPOULOU, et al., 2005).

Considerando a alta ocorrência de tumores mamários em cadelas e a importância de se estabelecer fatores que estimem o prognóstico, objetivou-se correlacionar o tamanho, tipo histológico e tempo de evolução de carcinomas mamários de 79 cadelas atendidas no Hospital Veterinário da Universidade Federal de Uberlândia.

\section{MATERIAL E MÉTODOS}

\section{Amostragem}

O estudo foi realizado no Hospital Veterinário da Universidade de Uberlândia, com aprovação da Comissão de Ética na Utilização de Animais da Universidade Federal de Uberlândia, protocolo 048/2014.

Foram utilizadas 79 cadelas encaminhadas voluntariamente pelos proprietários para avaliação clínica. As massas tumorais foram mensuradas em seu maior comprimento, com a utilização de um paquímetro, sendo posteriormente agrupados conforme o tamanho em T1 (tumores menores que $3 \mathrm{~cm}$ ), T2 (entre $3 \mathrm{~cm}$ a $5 \mathrm{~cm}$ ) e T3 (maiores que $5 \mathrm{~cm}$ ) (TNM, 1998).

Durante a avaliação clínica, questionou-se ao proprietário a respeito do tempo de evolução do tumor, sendo este o período compreendido entre a detecção da massa tumoral pelo proprietário e a exérese cirúrgica.

\section{Análise Histopatológica}

As cadelas com diagnóstico clínico de neoplasia mamária foram encaminhadas para exérese cirúrgica e fragmentos do tumor foram coletados para a realização do exame histopatológico. As amostras foram fixadas em formol 10\% por 48 horas, processadas rotineiramente para confecção de lâminas histológicas e coradas com Hematoxilina e Eosina. As lâminas foram analisadas por dois observadores que atribuíram classificação histológica conforme MISDORP et al., (1999). Em situações em que uma cadela apresentava mais de um tipo histológico de tumor, foi considerado o mais maligno. A partir dessa classificação, os animais foram agrupados em três diferentes grupos, sendo: carcinoma simples, quando havia apenas proliferação epitelial; carcinoma complexo, quando havia proliferação mioepitelial e epitelial; e carcinoma em tumor misto quando apresentava proliferação epitelial com metaplasia cartilaginosa, de acordo com KARAYANNOPOULOU, et al., (2005).

\section{Análise estatística}

A análise estatística foi realizada utilizando a ferramenta Action do programa Excel, a fim de verificar a presença ou não de correlação entre o tamanho da massa tumoral e o tempo de evolução, com base no teste Pearson. Foi também analisada a correlação entre o tipo histopatológico e o tamanho das massas tumorais, utilizando o teste de Chi-quadrado e Kruskal Wallis. 


\section{RESULTADOS E DISCUSSÃO}

Foram avaliadas 79 cadelas e dentre estas, o menor tumor aferido foi de 1 $\mathrm{cm}$ e o maior de $15 \mathrm{~cm}$ de diâmetro, obtendo uma média de $3,93 \mathrm{~cm}$. Resultados semelhantes foram encontrados por SIMON et al. (2009) que descreveram média de $3,5 \mathrm{~cm}$ e tamanho variando de $0,6 \mathrm{~cm}$ a $14 \mathrm{~cm}$ e PENA, et al. (2013) os quais analisaram 60 cadelas e relataram variação entre 0,5 e $14 \mathrm{~cm}$.

Quando agrupados conforme a classificação da Organização Mundial de Saúde (OMS) 47,43\% dos tumores pertenciam ao grupo T1 (menores que $3 \mathrm{~cm}$ ), $16,66 \%$ ao grupo T2 (entre $3 \mathrm{~cm}$ a $5 \mathrm{~cm}$ ) e $35,89, \%$ encontravam-se no grupo T3 (acima de $5 \mathrm{~cm}$ ). OLIVEIRA-FILHO, et al., (2010) estudando o tamanho tumoral de 1380 cadelas no Brasil descreveram resultados similares, sendo $49,4 \%$ nódulos $\mathrm{T} 1$, $19,1 \%$ eram T2 e 31,4\% eram T3. Por outro lado, PEÑA, et al., (2013) relataram $69,23 \%$ dos nódulos $\mathrm{T} 1,23,08 \%$ eram T2 e $8,33 \%$ foram classificados como T3 em um trabalho na Espanha. A maior prevalência de nódulos T1 pode ser explicada devido à maior preocupação do proprietário com relação à exérese precoce.

Entre os animais avaliados, em 65 deles foi informado o tempo de evolução, sendo o menor tempo de um mês e o maior de 60 meses, com 12,8 meses de média. OLIVEIRA, et al., (2003) relataram tempo médio de evolução ligeiramente menor, de 9,5 meses.

Dentre os nódulos classificados como T1, o tempo de evolução médio foi de 9,75 meses, enquanto nos tumores T2, 10,27 meses e no grupo T3 a média foi 18,5 meses. Nota-se que à medida que o tamanho do tumor aumenta, ocorre também o aumento do tempo de evolução (Gráfico 1). Ao aplicar o teste de Pearson observou-se um $p$-valor significativo ( $p=0,0148)$, porém a força da correlação foi de aproximadamente 0,301 indicando uma correlação fraca e um $R^{2}$ de 0,091 , ou seja, somente $9 \%$ das correlações podem ser previstas. A baixa força da correlação pode ser explicada pelo desvio padrão dos dados de tempo, em que dentro de uma mesma classificação de tamanho a variação do tempo de evolução era muito grande. O desvio padrão pode ser observado na Figura 1.

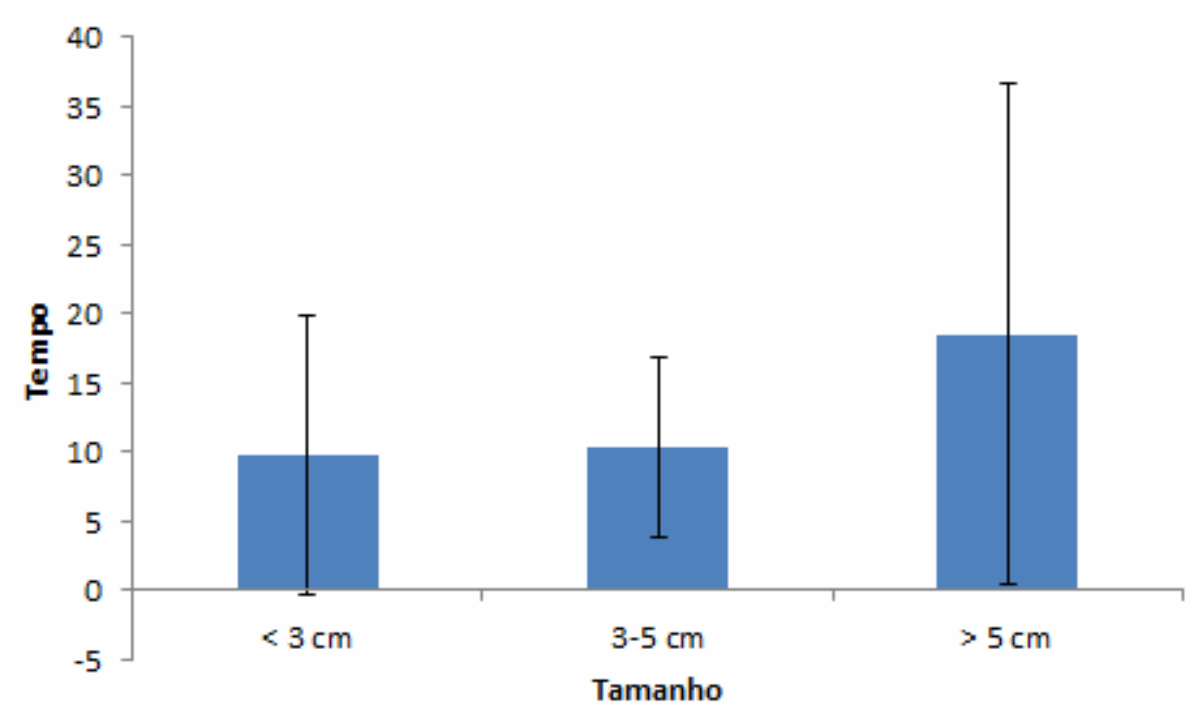

FIGURA 1 Média e Desvio Padrão do tempo de evolução de cadelas com neoplasia mamárias agrupadas de acordo com 0 tamanho das massas. 
Quanto ao tipo histopatológico, obteve-se um número variável dentro de cada tipo e quando agrupados de acordo com o tamanho (Tabela 1) não demonstraram concentração em nenhuma das classificações de tamanho. Segundo MATOS, et al., (2012) o tamanho dos tumores mamários está relacionado ao número de células em multiplicação e segundo SORENMO, et al., (2009) quanto maior for o tumor, maior tendência à malignidade. Aplicando a estatística de Chi-quadrado e Kruskal Wallis não foi obtido um p-valor significativo $(p=0,9)$ sugerindo que não há correlação entre o tamanho e o tipo de carcinoma, divergindo dos trabalhos já mencionados.

A falta de correlação entre o tipo histopatológico e o tamanho tumoral pode ser explicada devido à medida externa dos tumores não corresponderem ao tamanho real da neoplasia, uma vez que esta pode estar infiltrada nos tecidos adjacentes à glândula mamária assim como descrito por MATOS, et al. (2012). Outro problema na análise é o fato de que tumores maiores podem conter mais de um tipo histológico dentro da mesma massa, dificultando e por vezes mascarando o diagnóstico (ESTRELA-LIMA, et al., 2010).

TABELA 1 Tipos histopatológicos e tamanhos das massas tumorais de cadelas com neoplasia mamária.

\begin{tabular}{lccc}
\hline & \multicolumn{3}{c}{ Tamanho } \\
\cline { 2 - 4 } Tipo Histológico & $\mathbf{1 - 3 c m}$ & $\mathbf{3 - 5} \mathbf{c m}$ & $\mathbf{> 5 c m}$ \\
\hline Carcinoma simples & 18 & 8 & 11 \\
Carcinoma complexo & 8 & 2 & 4 \\
Carcinoma em tumor misto & 13 & 6 & 7 \\
Total & 39 & 16 & 22 \\
\hline
\end{tabular}

O estudo do tamanho tumoral apesar de ser visto como uma característica que pode influenciar no prognóstico, apresenta um valor subjetivo, uma vez que para a sua analise são necessárias ferramentas mais precisas para sua mensuração.

\section{CONCLUSÕES}

O trabalho mostrou que não há correlação entre o tamanho da massa tumoral e o tipo de carcinoma. O tamanho das neoplasias mamárias está susceptível a influência de diversas variáveis. Apesar da significância estatística mostrar que existe uma correlação fraca entre o tamanho e o tempo de evolução, existem fatores que distorcem o resultado sendo necessário o estudo das variáveis de forma mais sistemática e controlada.

\section{REFERÊNCIAS}

CASSALI, G. D.; LAVALLE, G. E.; DE NARDI, A. B.; FERREIRA, E.; BERTAGNOLLI, A. C.; ESTRELA-LIMA, A.; SOUZA, C. M. Consensus for the diagnosis, prognosis and treatment of canine mammary tumors. Brazilian journal of veterinary pathology, v. 4 n. 2, p. 153-180, 2013.

DE NARDI, A.B. Correlação da cicloxigenase-2 com Ki-67, p53 e Caspase-3 nas neoplasias de mama em cadelas. 2007. 92 f. Tese (Doutorado) - Faculdade de Ciências Agrárias e Veterinárias, Jaboticabal, São Paulo, 2007. 
DORÉ, M.; LANTHIER, I.; SIROIS, J. Cyclooxygenase-2 expression in canine mammary tumors. Veterinary Pathology, Filadelfia, v. 40, n. 2, p. 207-212, 2003.

ESTRELA-LIMA, A.; ARAÚJO, M. S.; COSTA-NETO, J. M.; TEIXEIRA-CARVALHO, A.; BARROUIN-MELO, S. M.; CARDOSO, S. V.; CASSALI, G. D. (2010). Immunophenotypic features of tumor infiltrating lymphocytes from mammary carcinomas in female dogs associated with prognostic factors and survival rates. BMC cancer, v. 10, n.1, p.256, 2010.

KARAYANNOPOULOU, M.; KALDRYMIDOU, E.; CONSTANTINIDIS, T. C.; DESSIRIS, A. Histological grading and prognosis in dogs with mammary carcinomas: application of a human grading method. Journal of comparative pathology, v. 133, n. 4, p. 246-252, 2005.

MARQUES, D.C.S. Avaliação da expressão da cox-2 em tumores mamários de cadela. 2013. 77f. Dissertação (Mestrado)- Faculdade de Medicina Veterinária Universidade de Lisboa, Lisboa, 2013

MATOS, A. J. F.; BAPTISTA, C. S.; GÄRTNER, M. F.; RUTTEMAN, G. R. Prognostic studies of canine and feline mammary tumours: the need for standardized procedures. The Veterinary Journal, v. 193, n. 1, p. 24-31, 2012.

MISDORP, WIM (Ed.). Histological classification of mammary tumors of the dog and the cat. Armed Forces Institute of Pathology: American Registry of Pathology: World Health Organization Collaborating Center for Comparative Oncology, 1999.

OLIVEIRA-FILHO, J. C., KOMMERS, G. D., MASUDA, E. K., MARQUES, B. M., FIGHERA, R. A., IRIGOYEN, L. F., BARROS, C. S. Estudo retrospectivo de 1.647 tumores mamários em cães. Pesquisa Veterinária Brasileira, v.30, n. 2, p. 177-185, 2010.

OLIVEIRA, L. O. D., OLIVEIRA, R. T. D.; LORETTI, A. P.; RODRGUES, R.; DRIEMEIER, D. Aspectos epidemiológicos da neoplasia mamária canina. Acta scientiae veterinariae. Porto Alegre, RS. Vol. 31, n. 2, p. 105-110, 2003.

OWEN, L. N. TNM classification of tumours in domestic animals. TNM classification of tumours in domestic animals., 1980.

PEÑA, L., DE ANDRES, P. J., Clemente, M., CUESTA, P., PEREZ-ALENZA, M. D. Prognostic Value of Histological Grading in Noninflammatory Canine Mammary Carcinomas in a Prospective Study With Two-Year Follow-Up Relationship With Clinical and Histological Characteristics. Veterinary Pathology Online, v. 50, n. 1, p. 94-105, 2013.

Petrov, E. A., ILIEVSKA, K., TROJACANEC, P., CELESKA, I., NIKOLOVSKI, G., GJUROVSKI, I., DOVENSKI, T. Canine mammary tumours-clinical survey. Macedonian Veterinary Review, v. 37, n. 2, p. 129-134, 2014. 
PINHO, S. S.; CARVALHO, S.; CABRAL, J. REIS, C. A. GARTNER, F. Canine tumors: a spontaneous animal model of human carcinogenesis. Translational Research, v. 159, n.3, p. 165-172, 2012.

SIMON, D.; SCHOENROCK, D.; NOLTE, II.; BAUMGARTNER ,W.; BARRON , R.; MISCHKE ,R. Cytologic examination of fine-needle aspirates from mammary gland tumors in the dog: diagnostic accuracy with comparison to histopathology and association with postoperative outcome. Veterinary Clinical Pathology. v. 38 p. 521525, 2009.

Sorenmo, K. U.; Kristiansen, V. M.; Cofone, M. A.; Shofer, F. S.; Breen, A. M.; Langeland, M.; Goldschmidt, M. H. Canine mammary gland tumours; a histological continuum from benign to malignant; clinical and histopathological evidence. Veterinary and comparative oncology, v. 7, n. 3, p. 162-172, 2009. 